\title{
Estimated intake of milk fat is negatively associated with cardiovascular risk factors and does not increase the risk of a first acute myocardial infarction. A prospective case-control study
}

\author{
Eva Warensjö ${ }^{1}$, Jan-Håkan Jansson ${ }^{2}$, Lars Berglund ${ }^{1}$, Kurt Boman ${ }^{2}$, Bo Ahrén ${ }^{3}$, \\ Lars Weinehall ${ }^{4}$, Bernt Lindahl ${ }^{5}$, Göran Hallmans ${ }^{6}$ and Bengt Vessby ${ }^{1 *}$ \\ ${ }^{1}$ Unit for Clinical Nutrition Research, Department of Public Health and Caring Sciences, \\ Uppsala University, Uppsala, Sweden \\ ${ }^{2}$ Department of Medicine-Geriatric, Skellefteå County Hospital, Umeå University, Umeå, Sweden \\ ${ }^{3}$ Department of Medicine, Lund University, Malmö, Sweden \\ ${ }^{4}$ Epidemiology, Department of Public Health and Clinical Medicine, Umeå University, Umeå, Sweden \\ ${ }^{5}$ Behavioral Medicine, Department of Public Health and Clinical Medicine, Umeå University, Umeå, Sweden \\ ${ }^{6}$ Nutritional Research, Department of Public Health and Clinical Medicine, Umeå University, Umeå, Sweden
}

(Received 23 May 2003 - Revised 26 November 2003 - Accepted 28 November 2003)

\begin{abstract}
Milk fat is high in saturated fatty acids (SFA) and high intakes of SFA are associated with cardiovascular diseases. The aim of the present study was to prospectively evaluate the potential risk of a first-ever acute myocardial infarction (AMI) in relation to the estimated milk-fat intake, reflected as the proportions of pentadecanoic acid $(15: 0)$ and heptadecanoic acid $(17: 0)$ in serum lipid esters. This was evaluated in a study population selected within the Västerbotten Intervention Program and the northern Sweden 'Monitoring of Trends and Determinants in Cardiovascular disease' survey populations. A prospective case-control design was used. The proportions of the biomarkers were lower in the cases $(n 78)$ than in the controls $(n 156)$, who were matched for age, sex, sampling time and geographical region. The standardised odds ratios of becoming an AMI case were between 0.7 and 0.8 for the biomarkers. The proportions of 15:0 and 17:0 in serum phospholipids were significantly and negatively correlated to serum concentrations of plasminogen activator inhibitor-1, tissue-type plasminogen activator, triacylglycerols, insulin, specific insulin, pro-insulin and leptin (all $P<0 \cdot 0001$ ), suggesting a negative relationship to the insulin-resistance syndrome and the risk of CHD. Adjustment for BMI did not materially change the relationships. Although there seems to be a negative association between milk-fat intake as mirrored by the proportions of 15:0 and 17:0 in serum lipid esters and a first-ever AMI, adjustment for clinical risk factors removed this relationship.
\end{abstract}

Pentadecanoic acid: Heptadecanoic acid: Milk fat: Acute myocardial infarction

Atherosclerosis and its consequences are currently the leading cause of death in the Western world (Libby, 2000). Many risk factors have been linked to the development of atherosclerotic cardiovascular disease including dietary fatty acid composition (Willett, 1998; Schaefer, 2002). A diet high in saturated fatty acids (SFA) and cholesterol has long been associated with the risk of cardiovascular disease (Keys et al. 1986; Ulbricht \& Southgate, 1991). The main mediating factor between dietary fat consumption and CHD risk is thought to be blood cholesterol concentration, which is directly affected by the dietary fatty acid composition. Other factors that probably link saturated fat consumption and CHD risk are atherosclerosis (long-term effects of diet), thrombosis (short-term effects of diet and hormonal responses to external events)
(Ulbricht \& Southgate, 1991), and insulin resistance (Vessby, 2000). The intake of milk and other dairy products contributes to a large proportion of the total and saturated fat intake (and of cholesterol as well), thus suggesting a link between the risk of cardiovascular disease and the consumption of dairy products.

It is known that the fatty acid composition in the blood and body tissues partly reflects the fatty acid composition of the diet at various time points after ingestion. Changes in the composition of dietary fatty acid intake are reflected in plasma lipids and erythrocyte membranes weeks and months after intake respectively, while the incorporation of fatty acids in adipose tissue reflects long-term changes in the diet (years) (Dougherty et al. 1987; Glatz et al. 1989; Ma et al. 1995; Katan et al. 1997). It has been

\footnotetext{
Abbreviations: ag, antigen; AMI, acute myocardial infarction; MONICA, Monitoring of Trends and Determinants in Cardiovascular disease; OR, odds ratio; PAI, plasminogen activator inhibitor; SFA, saturated fatty acid; $t$-, tissue-type.

* Corresponding author: Dr Bengt Vessby, fax +46 18611 7976, email bengt.vessby@pubcare.uu.se
} 
suggested that the fatty acid composition of serum lipid esters predicts CHD. An increased risk is associated with a higher proportion of SFA (Miettinen et al. 1982).

The fatty acids pentadecanoic acid $(15: 0)$ and heptadecanoic acid (17:0) in adipose tissue and serum phospholipids and cholesteryl esters have recently been proposed as biomarkers of dietary ruminant fat (milk fat and fat from ruminants; for example, beef and sheep) intake. Several studies have verified these as relative and objective estimates of milk-fat intake, both in men and women (Wolk et al. 1998, 2001; Smedman et al. 1999) in a Western population. Unlike ruminal microbes of dairy cattle, the human body is unable to synthesise fatty acids with an uneven number of $\mathrm{C}$ atoms (Wu \& Palmquist, 1991). To measure the serum content of 17:0 and/or 15:0 in serum lipid esters or the corresponding proportion of triacylglycerol fatty acid content of adipose tissue will consequently be a way to estimate the milk-fat intake. The term 'milk fat' includes all fat from dairy foods such as milk, cheese, ice cream, yoghurt and butter (Berner, 1993).

CHD mortality differences between countries were in a meta-analysis (forty countries) explained by differences in SFA and cholesterol intakes. Paradoxically, mortality rates were low in France and high in Finland, although intakes of SFA and cholesterol were comparable. It was suggested that this difference was due to a high consumption of dairy products in Finland and a high intake of plant foods in France respectively (Artaud-Wild et al. 1993). However, it has been difficult to demonstrate significant relationships between the estimated intake of saturated fat and the incidence of CHD in prospective studies within populations (Pietinen et al. 1997). This might be due to methodological problems; for example, day-to-day variations in individual intake as compared with differences between individual groups. Some intervention studies have demonstrated that dairy products may induce hypocholesterolaemic effects in human subjects (Eichholzer \& Stähelin, 1993), and adolescents with a high intake of dairy fat had low serum cholesterol levels (Samuelsson et al. 2001). Also, a recent study in the USA showed that dairy consumption was inversely related to manifestations of the insulin-resistance syndrome among young adults that were overweight from the beginning (Pereira et al. 2002).

It seems relevant to question whether a high consumption of dairy products is really harmful with respect to CHD in general and acute myocardial infarction in particular. The aim of the present study was to prospectively evaluate the potential risk of a first-ever AMI in relation to the estimated milk-fat intake, reflected as the proportions of 15:0 and $17: 0$ in serum lipid esters. The study was approved by the research ethics committee at Umeå University and all participants had given informed consent.

\section{Methods}

\section{Study population}

The study population was selected within the Västerbotten Intervention Program and the northern Sweden Monitoring of Trends and Determinants in Cardiovascular disease
(MONICA) survey populations, according to procedures as described earlier (Huhtasaari et al. 1988; Weinehall et al. 1998a,b). In the present prospective study, baseline is the day when cases and controls had participated in the respective health survey and had donated a blood sample to the Northern Sweden Medical Research Bank.

\section{Case finding}

AMI events were defined according to the MONICA project criteria (Anonymous, 1988) and incidence cases were defined by the northern Sweden MONICA incidence registry (Huhtasaari et al. 1988). At the time of definition, 30 September 1994, eighty-six cases fulfilled the following two criteria:

(1) The cases were registered in the northern Sweden MONICA incidence registry during the period between 1 January 1985 and 30 September 1994.

(2) The cases had participated in the health surveys before the AMI event and at the same time had donated a blood sample to the Northern Sweden Medical Research Bank.

Seventy-eight cases (sixty-two men and sixteen women) remained for analyses after the exclusion of six individuals with cancer and two individuals without blood samples. Two controls were randomly selected within the two survey populations and matched for sex, age ( \pm 2 years), type and date of health survey ( \pm 1 year) and geographical region. Potential controls were excluded if they had died or moved away from the area before 30 September 1994, or if a prior AMI, stroke or cancer diagnosis could not be excluded.

\section{Biochemical analyses}

Blood samples were collected after a minimum of $4 \mathrm{~h}$ fasting and analysed as described previously. During the first years of the Västerbotten Intervention Program health survey the requested fasting period was $4 \mathrm{~h}$. Since a majority of the health surveys were performed in the morning most participants had an overnight fast. The minimum fasting time was changed in 1992 to $8 \mathrm{~h}$ in the Västerbotten Intervention Program health survey. Cholesterol (Weinehall et al. 1998a,b), triacylglycerols and fasting plasma glucose were measured immediately after collection at each study centre using capillary blood, using Reflotron instruments (Boehringer Mannheim Scandinavia, Bromma, Sweden). All other measurements were performed on serum samples that were prepared at the most $2 \mathrm{~h}$ after collection and then frozen temporarily at $-20^{\circ} \mathrm{C}$ for up to 1 week. The samples were then transported to the blood bank and stored at $-80^{\circ} \mathrm{C}$ until analysed. The different measurements were carried out at several collaborating laboratories as described previously (except for fatty acid composition) (Thörgersen et al. 1998; Lindahl et al. 1999; Söderberg et al. 1999).

\section{Fatty acid composition of serum lipid esters}

The fatty acid analyses were performed at the clinical research laboratory at the Department of Public Health and Caring Sciences at Uppsala University. The fatty acid 
composition of the serum lipids was analysed by means of GLC, as described in detail elsewhere (Boberg et al. 1985). Briefly, the serum lipids were extracted in chloroform, separated by TLC, transmethylated and separated by GLC on a capillary column. The analyses were carried out on a GC 5890, equipped with a 7671A auto-injector, a 3392A integrator (all from Hewlett-Packard, Avondale, PA, USA) and a $25 \mathrm{~m}$ Nordion fused silica column NS-351 (HNU Systems Inc., Helsinki, Finland), using $\mathrm{He}$ as the carrier gas. The temperature was programmed to $100-210^{\circ} \mathrm{C}$. The fatty acids were identified by comparing each peak's retention time with those of methyl ester standards (GLC- 68A; $\mathrm{Nu}$ Check Prep, Elysian, MN, USA). The relative amount of each fatty acid (percentage of total fatty acids) was quantified by integrating the area under the peak and dividing the results by the total area for all fatty acids. The CV for the analyses were $<10 \%$ for all fatty acids in both phospholipids and cholesteryl esters, except for 15:0 in cholesteryl esters with a CV of $13.4 \%$.

\section{Biomedical analyses}

Blood pressures were recorded after $5 \mathrm{~min}$ of rest in a sitting or recumbent position (adjusted for sitting posture) using an $\mathrm{Hg}$ sphygmomanometer. The diastolic blood pressure was defined as Korotkoff's 5th phase. Hypertension was defined as systolic blood pressure $\geq 160 \mathrm{mmHg}$ and/or diastolic blood pressure $\geq 95 \mathrm{mmHg}$ or if the patient was on anti-hypertensive medication during a period of $14 \mathrm{~d}$ before the health survey. Smokers were divided into daily smokers and non-smokers. Smokers were those reporting a daily consumption of cigarettes, cigarillos, cigars or pipe tobacco. Individuals reporting occasional smoking or being an ex-smoker were included in the non-smokers group. History of diabetes was obtained from self-reported questionnaires. Weight was measured without shoes in light clothing to the nearest $0.2 \mathrm{~kg}$ and height to the nearest $10 \mathrm{~mm}$, without shoes. BMI was calculated as body weight $(\mathrm{kg})$ divided by height $\left(\mathrm{m}^{2}\right)$ (Thörgersen et al. 1998; Lindahl et al. 1999; Söderberg et al. 1999).

\section{Statistical analyses}

The statistical analyses were performed using the software packages STATA (version 6.0; STATA Corp., College Station, TX, USA), JMP (version 3.1; SAS Institute Inc., Cary, NC, USA) and SAS (version 6.12; SAS Institute Inc.) on a personal computer. The normal distribution of the continuous variables was examined with Shapiro-Wilk's test. Variables not normally distributed $(W<0.95)$ were log-transformed. The continuous variables were standardised and used in the conditional logistic regression analyses.

\section{Clinical risk profile and biomarkers}

For each clinical variable the risk of developing a firstever AMI was estimated as the standardised odds ratio (OR) by using univariate conditional logistic regression analysis. The clinical variables were BMI, systolic blood pressure, diastolic blood pressure, von Willebrandt factor, plasminogen activator inhibitor (PAI)-1 antigen (ag), Apo A-1, Apo B, triacylglycerols, cholesterol, tissue-type (t-) PA-ag, insulin, pro-insulin, specific insulin, leptin, plasma glucose, history of smoking, diabetes and hypertension. The same analyses were performed with 15:0 and 17:0 as predictor variables.

\section{Correlations between risk profile for developing acute myocardial infarction and estimated milk-fat intake}

Correlations between the proportions of $15: 0$ and $17: 0$, and their sum in serum phospholipids and clinical study variables, were explored with Pearson's rank correlation. The test was performed with and without adjustment for BMI. Further adjustment with BMI in combination with smoking history was made, as well. The proportions of biomarkers in serum phospholipids were used in the analysis since the predictor values were stronger than for those of the biomarkers in cholesteryl esters.

\section{Effect of biomarkers of milk-fat intake on the risk of developing acute myocardial infarction, when adjusted for clinical risk variables}

To calculate the OR of a first-ever AMI in relation to the proportions of $15: 0,17: 0$ and their sum in serum phospholipids, bivariate and multivariate logistic regression analyses were used. Adjustment with all clinical variables (as defined previously) taken together in a multivariate analysis was first made. Bivariate logistic regression analyses were then performed to establish OR for $15: 0,17: 0$, and $15: 0+17: 0$ in serum phospholipids to predict a first-ever AMI, after adjustment with specific clinical variables. Finally the logistic regression analyses were performed when adjusted for two different groups of CHD risk factors. The two groups were defined by us as 'classical' risk factors (cholesterol, blood pressures and smoking habits) and 'metabolic' risk factors (triacylglycerols, insulin, pro-insulin, t-PA, PAI-1, leptin and von Willebrandt factor).

The linearity assumption of the logistic regression models was checked by comparing the above-mentioned models to models where the biomarker $(15: 0+17: 0$ in serum phospholipids) with the strongest predictor value was dichotomised. This showed an attenuation of the relationships to AMI. This finding indicates that the relationship is approximately linear. All tests were twotailed and $P$ values $<0.05$ were considered significant.

\section{Results}

All seventy-eight cases (sixteen women and sixty-two men) and 156 control subjects (thirty-two women and 124 men) in the present study had participated in health surveys before the onset of their first AMI. The mean time between health survey and onset of disease was 18 months (median 15 months) (Weinehall et al. 1998a,b).

\section{Clinical risk profile}

The clinical characteristics of case and control groups at baseline are presented in Table 1. Any differences between case and control subjects are presented as standardised OR 
Table 1. Clinical baseline characteristics

(Mean values and standard deviations)

\begin{tabular}{|c|c|c|c|c|c|c|c|c|}
\hline \multirow[b]{2}{*}{ Variable } & \multicolumn{3}{|c|}{ Cases } & \multicolumn{3}{|c|}{ Controls } & \multicolumn{2}{|c|}{ Difference } \\
\hline & $n$ & Mean & SD & $n$ & Mean & SD & OR & $P$ \\
\hline Age (years) & 77 & $54 \cdot 8$ & $7 \cdot 2$ & 156 & $54 \cdot 6$ & $7 \cdot 2$ & & \\
\hline BMI $\left(\mathrm{kg} / \mathrm{m}^{2}\right)^{\star}$ & 72 & $27 \cdot 5$ & $4 \cdot 3$ & 156 & $25 \cdot 6$ & 3.7 & 1.64 & 0.001 \\
\hline Triacylglycerols (mmol/l) & 50 & $2 \cdot 64$ & $2 \cdot 70$ & 102 & $1 \cdot 71$ & $1 \cdot 20$ & 1.66 & 0.010 \\
\hline Cholesterol $(\mathrm{mmol} / \mathrm{l})^{\star}$ & 76 & $6 \cdot 80$ & 1.40 & 156 & $6 \cdot 40$ & $1 \cdot 20$ & 1.26 & 0.13 \\
\hline Apo A-1 (g/l) & 75 & 1.06 & $0 \cdot 17$ & 152 & $1 \cdot 15$ & $0 \cdot 17$ & 0.60 & 0.001 \\
\hline Aро B $(\mathrm{g} / \mathrm{l})$ & 75 & 1.21 & 0.27 & 152 & $1 \cdot 10$ & 0.27 & 1.43 & 0.014 \\
\hline Insulin (pmol/l) & 76 & $73 \cdot 2$ & $119 \cdot 9$ & 153 & $49 \cdot 8$ & $52 \cdot 8$ & 1.36 & 0.036 \\
\hline Pro-insulin (pmol/l) & 75 & $17 \cdot 6$ & $34 \cdot 2$ & 153 & $10 \cdot 8$ & $20 \cdot 0$ & 1.43 & 0.013 \\
\hline Specific insulin $(\mathrm{pmol} / \mathrm{l})^{\star}$ & 75 & $54 \cdot 0$ & $67 \cdot 0$ & 153 & 37.6 & 74.2 & 1.44 & 0.022 \\
\hline Plasma glucose (fasting) (mmol/l) & 58 & 5.04 & 0.67 & 117 & 5.07 & 0.62 & 1.08 & 0.670 \\
\hline Leptin (ng/ml) & 70 & $10 \cdot 5$ & $10 \cdot 4$ & 143 & 6.87 & $6 \cdot 20$ & 1.60 & 0.000 \\
\hline PAl-1-ag ( $(\mu \mathrm{l} / \mathrm{I})$ & 76 & $15 \cdot 6$ & $14 \cdot 0$ & 155 & $11 \cdot 2$ & $9 \cdot 1$ & 1.38 & 0.086 \\
\hline t-PA-ag $\left(\mu \mathrm{l} / \mathrm{l}^{\star}\right.$ & 75 & 12.5 & $5 \cdot 60$ & 156 & $9 \cdot 10$ & 4.0 & $2 \cdot 15$ & 0.000 \\
\hline Systolic blood pressure (mmHg) & 73 & $143 \cdot 2$ & $22 \cdot 4$ & 156 & $136 \cdot 6$ & 19.5 & 1.41 & 0.018 \\
\hline Diastolic blood pressure (mmHg) & 73 & 87.7 & $11 \cdot 0$ & 156 & $84 \cdot 2$ & $10 \cdot 5$ & 1.36 & 0.018 \\
\hline $\operatorname{vWF}\left(\%\right.$ of normal) ${ }^{*}$ & 74 & $146 \cdot 6$ & $47 \cdot 9$ & 150 & $131 \cdot 8$ & $45 \cdot 8$ & 1.43 & 0.003 \\
\hline Number of smokers & 67 & 29 & & 148 & 41 & & 1.94 & 0.023 \\
\hline Number of subjects with diabetes & 76 & 6 & & 156 & 1 & & 1.76 & 0.068 \\
\hline Number of subjects with hypertension & 76 & 31 & & 156 & 43 & & $2 \cdot 32$ & 0.001 \\
\hline
\end{tabular}

OR, univariate odds ratio; PAl-1-ag, plasminogen activator inhibitor-1 antigen; t-PA-ag, tissue-type plasminogen activator antigen; vWF, von Willebrandt factor.

${ }^{\star}$ Tests based on log-transformed values.

calculated for each individual clinical variable. When subjects with missing values of any of the variables (except triacylglycerols) were excluded, the analyses rendered values (data not shown) that were only slightly different from the OR shown in Table 1.

\section{Biomarker composition of serum lipids}

The proportions of the biomarkers $15: 0$ and $17: 0$ in serum lipid esters for cases and controls were as shown in Table 2. The means of the proportions were found to be slightly lower in the case group. However, significant differences in means based on univariate OR were observed only in a few cases as described in Table 2. The OR for each standard deviation increase of the biomarkers, in serum phospholipids and cholesteryl esters, were calculated separately for each fatty acid with data from all individuals.
If subjects with the missing values of any of the variables (except triacylglycerols) were excluded in a repeated analysis, OR were in the same range, but the significance attenuated for most variables and disappeared in a few cases. The proportions of other analysed fatty acids in serum lipids differed between cases and controls for EPA $(20: 5 n-3)$ and docosapentaenoic acid (22:5n-3). These were significantly higher for the control subjects than the case subjects. In order to determine if the associations between serum biomarkers and the risk of a first-ever AMI were mediated by the simultaneous high proportions of EPA and docosapentaenoic acid, adjusted OR were determined. The relationship did not change much, thus indicating that high proportions of marine fatty acids such as EPA and docosapentaenoic acid did not mediate the observed effects of the proportions of $15: 0$ and 17:0 in serum phospholipids.

Table 2. Proportions of pentadecanoic acid $(15: 0)$ and heptadecanoic acid $(17: 0)(\%)$ and the relative risk, for each standard deviation increase of the biomarkers in serum cholesteryl esters and phospholipids, calculated as the odds ratio of developing a first-ever acute myocardial infarction*

(Mean values and standard deviations)

\begin{tabular}{|c|c|c|c|c|c|c|c|c|c|}
\hline \multirow[b]{2}{*}{ Lipid } & \multicolumn{3}{|c|}{ Cases } & \multicolumn{3}{|c|}{ Controls } & \multicolumn{3}{|c|}{ Relative risk } \\
\hline & $n$ & Mean & SD & $n$ & Mean & SD & OR & $95 \% \mathrm{Cl}$ & $P$ \\
\hline \multicolumn{10}{|l|}{ Cholesteryl ester } \\
\hline $15: 0 \dagger$ & 67 & 0.21 & 0.045 & 141 & 0.22 & 0.053 & 0.79 & $0.59,1.07$ & NS \\
\hline $17: 0 \dagger$ & 64 & 0.11 & 0.025 & 128 & 0.12 & 0.025 & 0.77 & $0.57,1.04$ & NS \\
\hline $15: 0+17: 0 \dagger$ & 64 & 0.33 & 0.058 & 128 & 0.35 & 0.067 & 0.75 & $0.55,1.00$ & 0.053 \\
\hline \multicolumn{10}{|l|}{ Phospholipid } \\
\hline $15: 0 \dagger$ & 62 & 0.21 & 0.04 & 132 & 0.22 & 0.049 & 0.72 & $0.53,0.98$ & 0.036 \\
\hline $17: 0$ & 68 & 0.42 & 0.06 & 141 & 0.44 & 0.071 & 0.75 & $0.57,1.00$ & 0.047 \\
\hline $15: 0+17: 0 \dagger$ & 62 & 0.63 & 0.094 & 132 & 0.67 & 0.11 & 0.79 & $0.65,0.96$ & 0.020 \\
\hline
\end{tabular}

OR, odds ratio of standardised variables.

* For details of subjects and procedures, see Table 1 and p. 636

† Log-transformed values. 
Correlations between risk profile for developing acute myocardial infarction and estimated milk-fat intake

The correlations between the proportions of $15: 0+17: 0$ in serum phospholipids and clinical variables were generally negative and are presented in Table 3 . The significant correlations remained, but were somewhat weakened after adjustment with BMI for all variables, except for the von Willebrandt factor where the significance disappeared. Further adjustment with BMI in combination with smoking habits did not change the correlation coefficients much, but attenuated the statistical significance in most cases (data not shown).

Effect of biomarkers of milk-fat intake on the risk of developing acute myocardial infarction, when adjusted for clinical risk variables

Adjustment with all clinical risk factors (both 'classical' and 'metabolic') taken together in a multivariate analysis removed the significant effect of the fatty acids to predict a first-ever AMI (data not shown). The results from the bivariate regression analyses, taking specific risk factors into account, followed the pattern from the multivariate analyses with the two risk-factor groups, as will be discussed. The 'classical' risk factors, one by one, did not change the relationship much, but each individual 'metabolic' risk factor removed any effect (data not shown). The results after the two multivariate regression analyses when adjusted for the two CHD risk-factor groups were as shown in Table 4. Only data from the analyses with the biomarker with the strongest predictor value, $15: 0+17: 0$ in serum phospholipids, are shown. Adjustment for cholesterol, blood pressures and smoking habits removed the statistical significance, which might be explained by the smaller number of subjects in the analysis due to missing data. However, there was virtually no change of the OR to predict a first-ever AMI (OR 0.82v. 0.83). Adjustment for 'metabolic' risk factors removed any relationship $(\mathrm{OR} \geq 1 \cdot 0)$.

\section{Discussion}

The findings in the present prospective case-control study, in which the AMI cases were defined within two health survey populations, suggest that a higher intake of fat from dairy products, as estimated from the proportions of

Table 3. Pearson correlation coefficients $(r)$ between the sum of pentadecanoic acid $(15: 0)$ and heptadecanoic acid $(17: 0)$ in serum phospholipids and clinical variables, with and without adjustment for body mass index

\begin{tabular}{|c|c|c|c|c|}
\hline \multirow[b]{3}{*}{ Clinical variable } & \multicolumn{4}{|c|}{ Phospholipid } \\
\hline & \multicolumn{2}{|c|}{$15: 0+17: 0^{*}$} & \multicolumn{2}{|c|}{$15: 0+17: 0^{\star}$ (BMI adjusted) } \\
\hline & $r$ & $P$ & $r$ & $P$ \\
\hline Triacylglycerols* & -0.40 & $<0.0001$ & -0.30 & $<0.001$ \\
\hline Cholesterol & -0.16 & $<0.05$ & -0.15 & $<0.05$ \\
\hline Apo A-1 & 0.04 & NS & -0.04 & NS \\
\hline Aро B & -0.07 & NS & -0.04 & NS \\
\hline Insulin* & -0.42 & $<0.0001$ & -0.27 & $<0.001$ \\
\hline Pro-insulin* & -0.40 & $<0.0001$ & -0.27 & $<0.001$ \\
\hline Specific insulin* & -0.36 & $<0.0001$ & -0.21 & $<0.001$ \\
\hline Leptin* & -0.36 & $<0.0001$ & -0.20 & $<0.05$ \\
\hline PAl-1-ag* & -0.48 & $<0.0001$ & -0.36 & $<0.0001$ \\
\hline t-PA-ag* & -0.40 & $<0.0001$ & -0.27 & $<0.001$ \\
\hline Systolic blood pressure & -0.08 & NS & 0.001 & NS \\
\hline Diastolic blood pressure & -0.06 & NS & 0.031 & NS \\
\hline $\mathrm{vWF}^{\star}$ & -0.18 & $<0.05$ & -0.12 & NS \\
\hline BMI & -0.35 & $<0.0001$ & & \\
\hline
\end{tabular}

* Based on log-transformed values.

PAl-1-ag, plasminogen activator inhibitor-1 antigen; t-PA-ag, tissue-type plasminogen activator antigen; vWF, von Willebrandt factor

Table 4. Univariate and multivariate conditional logistic regression: effect of pentadecanoic acid (15:0) + heptadecanoic acid $(17: 0)^{\star}$ in serum phospholipids on acute myocardial infarction, before and after adjustment for 'classical' and 'metabolic' risk factors

\begin{tabular}{lcccr}
\hline Covariate* $^{*}$ & OR & $P$ value & $95 \%$ Cl & No. of observations \\
\hline None & 0.79 & 0.020 & $0.65,0.96$ & 195 \\
None† & 0.82 & 0.040 & $0.67,0.99$ & 178 \\
Classical risk factors $\ddagger$ & 0.83 & 0.087 & $0.67,1.03$ & 168 \\
Metabolic risk factors§ & 1.038 & 0.826 & $0.74,1.46$ & 114 \\
\hline
\end{tabular}

* Standardised variables.

† Subjects with missing values of triacylglycerols excluded.

$\ddagger$ Classical risk factors are cholesterol, smoking habits, systolic blood pressure and diastolic blood pressure.

$\S$ Metabolic risk factors are triacylglycerols, specific insulin, insulin, pro-insulin, BMI, leptin, plasminogen activator inhibitor-1, tissue-type plasminogen activator and von Willebrandt factor. 
$15: 0$ and $17: 0$ and their sum $(15: 0+17: 0)$ in serum phospholipids (and cholesteryl esters), seems to be associated with a reduced, rather than an increased, risk of developing AMI.

The study population consisted of both men and women. We know that there might be cardiovascular risk-factor differences between men and women (Brochier \& Arwidson, 1998). However, repeating the same analyses on a group consisting of only the men gave only slightly different results, compared with the entire study population. Given this, data from the analyses including both men and women are shown and discussed in the present paper. The conditional logistic regression analyses were carried out on the subgroup of individuals (212 subjects) without any missing values of all variables, except the triacylglycerols. Excluding individuals with missing triacylglycerols values would have reduced the already low number of individuals in the analyses to 139 . The concentration of triacylglycerols is an important marker of insulin resistance and thereby also an important factor in the development of cardiovascular disease, but insulin resistance is related to a clustering of risk factors (Gensini et al. 1998), many of which were included in the analyses. Thus including some individuals with missing triacylglycerols values in our analyses anyway will probably not influence the outcome of the study.

The observed differences in clinical characteristics between the case and control groups at baseline were as expected, with a higher mean BMI and an increased frequency of lipid disorders and other metabolic abnormalities among the cases. The number of subjects with high blood pressures, diabetes and the reported number of smokers were greater among the cases than among the controls (Thörgersen et al. 1998; Lindahl et al. 1999; Söderberg et al. 1999). The case subjects had slightly lower proportions of 15:0 and 17:0 in the blood lipids, than did the control subjects. This suggests that a higher estimated consumption of dairy products does not predict a firstever AMI. Our analyses also rendered OR between 0.7 and 0.8 for the biomarkers, which suggests a $20-30 \%$ decreased risk of AMI after each standard deviation increase of the proportion of biomarkers, measured in blood serum phospholipids. The negative associations found between the proportions of biomarkers and the magnitude of other CHD risk factors might indicate that those with a high intake of dairy fat have a reduced rather than an increased associated risk. The factors found to most strongly confound the effect of milk-fat biomarkers on AMI (Table 4) were the risk factors defined by us as 'metabolic', such as PAI-1-ag, t-PA-ag, leptin and triacylglycerols, all closely associated with the metabolic impairment characteristic of the insulin-resistance syndrome (Haffner, 1996; Juhan-Vague et al. 1996). The 'classical' CHD factors did, however, not remove the effect of milk fat to predict a first-ever AMI, as the OR remained virtually unchanged. This observation might suggest that the positive effects of milk fat on the risk of AMI is separate from the effects of the 'classical' CHD risk factors, but correlated to 'metabolic' risk factors. We observed in the logistic regression model that the effect of the estimated intake of milk fat on AMI is removed, when adjusted for other 'metabolic' risk factors (Table 4). It is conceivable that high proportions of milk fat in the food, as reflected by the proportions of 15:0 and 17:0 in the blood, could affect the incidence of AMI through the effects on 'metabolic' risk factors. However, based on these results we can say nothing about the causality of the relationship. We can say, however, that the relationship between milk fat and AMI is not directly related to and not mediated by 'classical' risk factors in the subjects in the present study. Another possible explanation is that a high consumption of dairy fat is part of a 'healthy food pattern', thus reducing the risk of CHD and AMI.

The proportions of $15: 0$ and $17: 0$ in serum lipids and in adipose tissue triacylglycerols have in several studies been shown to be valid markers for dairy-fat intake (Wolk et al. 1998, 2001; Smedman et al. 1999). Accounting for the estimated intake of the same fatty acids in ruminant meat does not affect the relationships significantly (Wolk et al. 1998, 2001). A small proportion of $15: 0$ and 17:0 is also found in fat from fish but the absolute dietary intake is almost negligible, considering the low daily intake of fat from fish compared with that from dairy fat. The inverse relationship between the proportions of 15:0 and 17:0 in serum phospholipids and AMI in the present study is not confounded by the intake of fat from fish. The relationships were unchanged when adjusted for the proportions of EPA and docosahexaenoic acid, which are known to be valid markers for dietary intake of fat from fish.

\section{Biomarkers and risk factors in the development of acute myocardial infarction}

The significant and negative correlations we found between clinical variables and biomarkers in serum phospholipids confirm and extend previous findings among elderly men (Smedman et al. 1999). In that study, negative associations were found between biomarkers and body weight, BMI, Apo B, hip circumference and waist circumference, whereas positive correlations were found for Apo A-1 and HDL-cholesterol (Smedman et al. 1999). In a recent case-control study from Norway (Yli-Jama et al. 2002), patients with a first myocardial infarction were compared with population controls with regard to the pattern of serum non-esterified fatty acids. There was a decreasing risk of myocardial infarction with increasing quartiles of myristic acid (14:0), which is also a marker of milk-fat intake. Also the proportions of long-chain $n-3$ fatty acids, as well as of stearic acid (18:0), were found to be inversely related to the risk for myocardial infarction.

\section{Milk and milk products as markers of a 'healthy food pattern'?}

It is possible that a high intake of dairy products in the present study population is part of a 'healthy food pattern'; for example, a high consumption of fruits and vegetables. Such a diet is high in dietary fibre, vitamins and minerals. It is widely recognised that soluble dietary fibres favourably reduce cholesterol levels by yet unknown mechanisms. Dietary fibre may also change serum levels of certain hormones involved in lipid metabolism, thus affecting the 
development of CHD (van Horn, 1997). If the associations between milk-fat biomarkers and the development of AMI were a result of a 'healthy food pattern' we could expect the associations to disappear after adjustment for intakes of fruits, vegetables and wholegrain cereals. This adjustment was not done in the present study but was performed in another study, where negative correlations between $15: 0$ in serum cholesteryl esters and metabolic variables were observed in young adolescents in Sweden. The adjustment had only a minor effect on the correlations (Samuelsson et al. 2001), indicating that fruit and vegetables (independent of energy intake) did not mediate the beneficial effects of the estimated intake of milk fat in that study. This is also supported by Smedman et al. (1999), where the negative associations observed between clinical variables and 15:0 in serum lipid esters remained, although in most cases weakened, after adjustment for, for example, potatoes, beet crops and vegetables. This is further supported by the study by Pereira et al. (2002). They found that the inverse correlation between dairy intake and the insulin-resistance syndrome was not confounded by dietary fibre intake (Pereira et al. 2002).

\section{'Metabolic' risk factors and milk fat}

'Metabolic' risk factors, such as PAI-ag, t-PA-ag, triacylglycerols, insulin, pro-insulin, specific insulin and leptin were all negatively correlated to the estimated milk-fat intake in the present study population. These variables are linked to the development of CHD and atherothrombotic disease (Gensini et al. 1998; Thörgersen et al. 1998; Lindahl et al. 1999; Söderberg et al. 1999). The clustering of risk factors increases the risk of CHD and is associated with insulin resistance (Solomon \& Manson, 1997; Gensini et al. 1998). An increased proportion of SFA in the diet impairs insulin sensitivity (Vessby et al. 2001). It is thus easy to believe that a diet rich in milk fat, and therefore high in SFA, would predict a first-ever AMI, but the results of the present study indicate the opposite. Many of the variables found to be negatively correlated to the estimated intake of milk fat are linked to obesity and insulin resistance. It is interesting that many of the negative associations between dairy-fat intake and 'metabolic' risk factors remained after adjustment for BMI, which might indicate that these associations are related to regional adiposity (central obesity), often consistent with the insulin-resistance syndrome, rather than to overweight as such (Haffner, 1996). Impaired fibrinolysis is also a common feature of the insulin-resistance syndrome and several thrombotic diseases such as AMI. PAI-1 has been found to be associated with many metabolic risk factors that may affect the risk of CHD such as BMI, central body fat and serum triacylglycerols and has been suggested to be a possible link between insulin resistance and CHD (Haffner, 1996). Mennen et al. (1999) found t-PA-ag to be inversely correlated with the intake of milk, milk products and cheese, independently of each other. These associations remained after adjustment for smoking, BMI, blood pressure and total cholesterol. They concluded that a high intake of dairy products may enhance fibrinolysis (Mennen et al. 1999).

\section{Conclusion}

Although there seems to be a negative association between a high milk-fat intake mirrored by the proportions of $15: 0$ and 17:0 in serum lipid esters, and a first-ever AMI, adjustment for clinical risk factors removed this relationship. We can speculate about possible mechanisms behind these findings, but it is not possible to draw any conclusions about causality. Further studies are needed to elucidate the mechanisms behind these relationships and to verify the findings of the present study.

\section{Acknowledgements}

Financial support was provided by the Swedish Medical Research Council (grant no. 27X-13083), the Swedish Heart and Lung Association and the Swedish Dairy Association.

\section{References}

Anonymous (1988) The World Health Organization MONICA Project (monitoring trends and determinants in cardiovascular disease): a major international collaboration. WHO MONICA Project Principal Investigators. J Clin Epidemiol 41, 105-114. Artaud-Wild SM, Connor SL, Sexton G \& Connor WE (1993) Differences in coronary mortality can be explained by differences in cholesterol in saturated fat intakes in 40 countries but not in France and Finland. Paradox. Circulation 88, 2771-2779.

Berner LA (1993) Roundtable discussion on milkfat, dairy foods, and coronary heart disease risk. J Nutr 123, 1175-1184.

Boberg M, Croon LB, Gustafsson IB \& Vessby B (1985) Platelet FA-composition in relation to fatty acid composition in plasma and to serum lipoprotein lipids in healthy subjects with specific reference to the linoleic acid pathway. Clin Sci $\mathbf{6 8}$, 581-587.

Brochier ML \& Arwidson P (1998) Coronary heart disease risk factors in women. Eur Heart $J$ 19, A45-A52.

Dougherty RM, Galli C, Ferro-Luzzi A \& Iacono JM (1987) Lipid and phospholipid fatty acid composition of plasma, red blood cells, and platelets and how they are affected by dietary lipids: a study on normal subjects in Italy, Finland and the USA. Am J Clin Nutr 45, 443-445.

Eichholzer M \& Stähelin H (1993) Is there a hypocholesterolemic factor in milk products? Int J Vitam Nutr Res 63, 159-163.

Gensini GF, Comeglio M \& Colella A (1998) Classical risk factors and emerging elements in the risk profile for coronary artery disease. Eur Heart $J$ 19, A53-A61.

Glatz JFC, Soffers EMF \& Katan MB (1989) Fatty acid composition of serum cholesteryl esters and erythrocyte membrane as indicators of linoleic intake in man. Am J Clin Nutr 49, 269-276.

Haffner SM (1996) Cardiovascular risk factors and the prediabetic syndrome. Ann Med 28, 363-370.

Huhtasaari F, Asplund K \& Wester PO (1988) Cardiovascular risk factors in the Northern Sweden MONICA Study. Acta Med Scand 224, 99-108.

Juhan-Vague I, Pyke S, Alessi MC, Jespersen J, Haverkate F \& Thompson SC (1996) Fibrinolytic factors and the risk of myocardial infarction or sudden death in patients with angina pectoris. ECAT Study Group. European Concerted Action on Thrombosis and Disabilities. Circulation 94, 2057-2063.

Katan MB, Deslypere JP, van Birgelen APJM, Penders M \& Zegwaard M (1997) Kinetics of the incorporation of dietary 
fatty acids into serum cholesteryl esters, erythrocyte membranes, and adipose tissue: an 18-month controlled study. J Lipid Res 38, 2012-2022.

Keys A, Menotti A, Karvonen MJ, Aravanis C, Blackburn C, Buzina R, Djordjevic BS, Dontas AS, Fidanza F \& Keys MH (1986) The diet and 15-year death rate in the seven countries study. Am J Epidemiol 124, 903-915.

Libby P (2000) Changing concepts of atherogenesis. J Intern Med 247, 349-358.

Lindahl B, Dinesen B, Eliasson M, Røder M, Jansson JH, Huhtasaari F \& Hallmans G (1999) High proinsulin concentration precedes acute myocardial infarction in a nondiabetic population. Metabolism 48, 1197-1202.

Ma J, Folsom A, Shahar E \& Eckfeldt JH (1995) Plasma fatty acid composition as an indicator of habitual dietary fat intake in middle-aged adults. Am J Clin Nutr 62, 565-571.

Mennen L, Balkau B \& Vol S (1999) Tissue-type plasminogen activator antigen and consumption of dairy products. The DESIR study. Data from an Epidemiological Study on Insulin Resistance Syndrome. Thromb Res 94, 381-388.

Miettinen TA, Naukkarinen JK, Huttunen S, Mattila S \& Kumlin T (1982) Fatty-acid composition of serum lipids predicts myocardial infarction. Br Med J 285, 993-996.

Pereira MA, Jacobs DR, Van Horn L, Slattery ML, Kartashov AI \& Ludwig DS (2002) Dairy consumption, obesity, and the insulin resistance syndrome in young adults. The CARDIA study. JAMA 287, 2081-2089.

Pietinen P, Ascherio A, Korhonen P, Hartman AM, Willett WC, Albanes D \& Virtamo J (1997) Intake of fatty acids and risk of coronary heart disease in a cohort of Finnish men. The Alpha-Tocopherol, Beta-Carotene Cancer Prevention Study. Am J Epidemiol 145, 876-887.

Samuelsson G, Bratteby LE, Mohsen R \& Vessby B (2001) Dietary fat intake in healthy adolescents. Inverse relationships between the estimated intake of saturated fatty acids and serum cholesterol. Br J Nutr 85, 333-341.

Schaefer EJ (2002) Lipoproteins, nutrition and heart disease. Am J Clin Nutr 75, 191-212.

Smedman A, Gustafsson IB, Berglund L \& Vessby B (1999) Pentadecanoic acid in serum as a marker for intake of milk fat: relations between intake of milk and metabolic risk factors. Am J Clin Nutr 69, 22-29.

Söderberg S, Ahrén B, Jansson JH, Johnson O, Hallmans G, Asplund K \& Olsson T (1999) Leptin is associated with increased risk of myocardial infarction. $J$ Intern Med 246, 409-418.

Solomon CG \& Manson JE (1997) Obesity and mortality: a review of epidemiological data. Am J Clin Nutr 66, 1044S-1050S.

Thörgersen AM, Jansson JH, Boman K, Nilsson TK, Weinehall L, Huhtasaari F \& Hallmans G (1998) High plasminogen activator inhibitor and tissue plasminogen activator levels in plasma precede a first acute myocardial infarction in both men and women. Evidence for the fibrinolytic system as an independent primary risk factor. Circulation 98, 2241-2247.

Ulbricht TLV \& Southgate DAT (1991) Coronary heart disease: Seven dietary factors. Lancet 338, 985-992.

van Horn L (1997) Fiber, lipids and coronary heart disease. Circulation 95, 2701-2704.

Vessby B (2000) Dietary fat and insulin actions in humans. Br J Nutr 83, Suppl. 1, S91-S96.

Vessby B, Unsitupa K, Hermansen K, et al. (2001) Substituting dietary saturated for monounsaturated fat impairs insulin sensitivity in healthy men and women: the KANWU Study. Diabetologia 44, 312-319.

Weinehall L, Hallgren CG, Westman G, Janlert U \& Wall S (1998a) Reduction of selection bias in primary prevention of cardiovascular disease through involvement of primary health care. Scand J Prim Health Care 16, 171-176.

Weinehall L, Johnsson O, Jansson JH, Boman K, Huhtasaari F, Hallmans G, Dahlen G \& Wall S (1998b) Perceived health modifies the effect of biomedical risk factors in the prediction of acute myocardial infarction. An incident case-control study from northern Sweden. J Intern Med 243, 99-107.

Willett W (1998) Diet and coronary heart disease. In Nutritional Epidemiology, Chapter 15, pp. 414-466 [W Willett, editor]. New York: Oxford University Press.

Wolk A, Vessby B, Ljung H \& Barrefors P (1998) Evaluation of a biological marker of dairy fat intake. Am J Clin Nutr $\mathbf{6 8}$, 291-295.

Wolk A, Furuheim M \& Vessby B (2001) Fatty acid composition of adipose tissue and serum lipids are valid biomarkers of dairy fat intake among men. $J$ Nutr 13, 828-833.

Wu Z. \& Palmquist D.L. (1991) Synthesis and biohydrogenation of fatty acids by ruminal microorganisms in vitro. $J$ Dairy $S c i$ 74, 3035-3046.

Yli-Jama P, Meyer HE, Ringstad J \& Pedersen JI (2002) Serum free fatty acid pattern and risk of myocardial infarction: a case-control study. J Intern Med 251, 19-28. 